\title{
PRESENTACIÓN DEL PROBLEMA BAJO UNA METODOLOGÍA REFLEXIVA PARA LA INVESTIGACIÓN CIENTÍFICA
}

\author{
PRESENTATION OF THE METHODOLOGY THAT HAS A PROBLEM UNDER A \\ REFLECTION FOR SCIENTIFIC RESEARCH \\ Juan Guillermo Miñano Lecaros* \\ Docente Principal de la Facultad de Ciencias Contables
}

Universidad Nacional Mayor de San Marcos-UNMSM / Lima-Perú

[Recepción: Setiembre de 2014/ Conformidad: Octubre 2014]

\section{RESUMEN}

La aplicación de la metodología reflexiva en la propuesta de un proyecto de tesis para optar el grado académico de magíster o doctor, debe expresar en su título, las variables que permitan desarrollar la investigación, el cual debe estar circunscrito al problema y entorno donde se halla dicho problema, y que mediante la aplicación de instrumentos epistemológicos y metodológicos de carácter objetivo, pueda plantearse la alternativa de solución. Para ello, la conceptualidad del investigador debe, al menos elegir un problema que afecta la gestión institucional en el plano donde se desenvuelve como profesional contable o de otra profesión afín a las Ciencias Contables, como una concepción para la propuesta de un título del proyecto en investigación.

\section{Palabras clave:}

Problema; entorno; materialismo; realidad objetiva; conciencia.

\begin{abstract}
The application of reflective proposed methodology in a project for thesis to obtain the academic degree of master's or doctor, must be expressed in its title, the variables to develop research, which should be confined to the problem and environment where is the problem, and that by applying epistemological and methodological tools of an objective character, can consider the alternative solution. For this, the conceptuality of the researcher should at least choose a problem that affects the governance in the plane where it operates as a professional accountant or other profession affined to Accounting, as a concept for a proposed project title research.
\end{abstract}

\section{Keywords:}

Problem; environment; materialism; objective reality; conscience.

\footnotetext{
* Doctor en Ciencias Contables y Empresariales, Magíster en Auditoría - UNMSM. E-mail: minano1969@hotmail.com
} 


\section{INTRODUCCIÓN}

La Resolución de Decanato Nº03/FCC-D/13 de la facultad de Ciencias Contables - UNMSM vigente a la fecha, prescribe que en los estudios de Maestría, se precisa los objetivos de los cursos del área de investigación, los cuales son los siguientes: en el primer semestre, el curso de "Metodología de la Investigación” tiene como objetivo la instrucción para los procesos de investigación; en el segundo semestre, el curso de "Seminario de Investigación Temática" tiene como objetivo final, la presentación del proyecto de tesis ante la Unidad de Posgrado; en el tercer semestre, el curso de "Investigación Aplicada - Tesis I" que tiene como objetivo la presentación del borrador de Tesis y en el cuarto semestre, por intermedio del curso de "Investigación Aplicada - Tesis II", el objetivo final es la actualización del borrador de tesis.

Asimismo, para los estudiantes del Doctorado, se precisa los estudios del área de investigación y sus correspondientes objetivos, según se detalla: El primer semestre, el curso de "Tesis I" tiene como objetivo la instrucción para los procesos de investigación; en el segundo semestre, mediante el curso de "Tesis II" su objetivo final es la presentación del proyecto de tesis ante la Unidad de Posgrado; en el tercer semestre, el curso de "Tesis III" tiene como objetivo final la presentación del borrador de tesis y en el cuarto y último semestre, el curso de "Seminario de Tesis Doctoral" que tiene como objetivo final la actualización y perfeccionamiento del borrador de tesis.

Como se podrá apreciar, la actual política académica en la Unidad de Posgrado, se circunda a que durante los dos años de estudios de Maestría y Doctorado, se debe elaborar y presentar el proyecto de tesis para ser evaluado e inscrito. Asimismo, elaborar el borrador de tesis a la conclusión de sus estudios de Posgrado.

Para el cumplimiento de dicho propósito, proponemos una metodología de carácter reflexiva, a fin de poder construir el respectivo título de investigación y luego, continuar con el desarrollo de la estructura del proyecto de tesis, tal como lo prescribe la Resolución Directoral N 080-EPG-2010 de la Escuela de Posgrado de la UNMSM, la misma que aprueba la "Directiva para el procedimiento de la elaboración de la Tesis para la obtención del grado de Magíster o Doctor".

\section{CONCEPTUALIDAD FILOSÓFICA DE LA INVESTIGACIÓN}

La investigación a realizarse, estriba básicamente en la percepción objetiva y materialista del investigador, del espacio real que lo rodea y es, en última instancia, el entorno donde desarrolla y aplica sus conocimientos, lo que permite detectar el problema a investigarse. Es por ello, que el "materialismo como certidumbre espontánea de todas las personas en la existencia objetiva del mundo exterior distingue la concepción del mundo que representa el desarrollo científico y; a su vez, refleja la realidad objetiva y material", tal como lo define el diccionario filosófico Rosental-Iudin (1976). En ese orden de acciones, la investigación debe orientarse a lo real, objetivo y descriptible del espacio que nos rodea. Ello, debe reflejarse en nuestro entorno, que para algunos investigadores será su centro laboral en el cual desempeñan una función principal o en el caso de los investigadores que son profesionales independientes, la elección de un entorno a la cual brindan sus servicios profesionales.

Definido la concepción filosófica, el investigador podrá interrelacionar el entorno y problema.

\section{EL ENTORNO}

Como espacio en el cual se aprecia objetivamente las interrelaciones de los sujetos, es para nuestro presente modelo epistemológico, la institución o ente en la cual se desarrolla una dinámica de gestión pública o privada. La administración de los recursos humanos, financieros y materiales se reflejan realmente en ese espacio, lo cual su carácter objetivo se aprecia realmente en lo expresado como gestión.

Las administraciones públicas como privadas, de una manera u otra, expresan problemas de gestión. $Y$ es inexorablemente, la necesidad objetiva y real de resolver dichos problemas, para lo cual el entorno expresa la diversidad de problemas que el sujeto, asociado a su conocimiento lo detecta como tal. 


\section{EL PROBLEMA DE LA INVESTIGACIÓN}

La percepción como resultado de la observación integral de las cosas reales y circundantes, permite al sujeto, apreciar problemas relacionados a su capacidad cognoscitiva, donde lo sensorial, refleja las cosas en la conciencia mediante los sentidos. Es por ello, que la aplicación de la observación para detectar el problema de investigación está relacionado al grado de la acumulación de conocimientos. La detección del problema está indisolublemente ligada al concepto realidad-conocimiento.

\section{LA EXPOSICIÓN DE VARIABLES - EL TÍTULO DE LA INVESTIGACIÓN CIENTÍFICA}

El método reflexivo exige la determinación de la variable independiente que refleja una característica axiomática de la observación y donde el sistema expositivo está referido al problema, que es real, objetivo y de carácter demostrable. Dicho problema, es hallado en un entorno, un espacio real que asume la definición de variable dependiente, pues en ella interrelacionan los sujetos entre sí, y que para la aplicación de nuestro método, es definido mediante el título del problema de investigación; por ejemplo, el siguiente proyecto de investigación para optar el Grado de Magíster en la Facultad de Ciencias Contables - UNMSM, titulado:"El problema de liquidez y rentabilidad en la gestión de la Sociedad de Beneficencia Pública del Callao-Perú 2008-2013”, expresa perfectamente las dos variables, independiente: "el problema de liquidez y rentabilidad" y la dependiente:"Sociedad de Beneficencia Pública del Callao", a ello, se agrega que en primer orden, el autor es funcionario de la referida entidad; así como, su formación profesional y experiencia en asuntos financieros, le ha permitido detectar el problema de investigación, lo que obviamente generará la correspondiente propuesta de alternativa de solución.

\section{METODOLOGÍA APLICABLE A LA INVESTIGACIÓN}

Consideramos como método no experimental, asociado a la investigación en Ciencias Contables, por lo que las unidades de análisis serán organiza- das para su evaluación. Por ejemplo, para asuntos de gobierno, las unidades de análisis lo conformará las leyes y regulaciones; así como, los instructivos y directivas contables, agregando a ello, las Normas Internacionales de Contabilidad para el sector público. En el siguiente trabajo de investigación titulado: "Propuesta de un modelo de control presupuestal en el gasto corriente; caso unidad ejecutora 003-Ejercito Peruano", se aprecia que el investigador es conocedor de dicha problemática institucional, lo que permite desarrollar la propuesta de un modelo de gestión, partiendo del análisis regulatorio en el ámbito normativo, doctrinario y contable sobre la gestión presupuestaria, más aún siendo miembro de la referida institución pública; todo ello es factible por el conocimiento y experiencia profesional del investigador, el cual destaca en la presentación del título del problema de investigación.

\section{CONCLUSIONES}

1. La construcción del título del proyecto de investigación, debe estar asociado indisolublemente a la percepción de un problema de la realidad objetiva y material.

2. La concepción materialista de las cosas de la realidad circundante permite una observación real y coherente.

3. La acumulación de conocimientos en el sujeto, permite un alto grado de desarrollo de la conciencia, lo que permite una percepción de la realidad de forma reflexiva.

4. La variable independiente es la expresión epistemológica del problema real y existente, que a su vez, tiene un impacto socio-económico en nuestra sociedad.

5. La variable dependiente, que es el entorno real y existente, permite ser evaluado en su plano de gestión y; a su vez, genera la propuesta de alternativa de solución al problema detectado.

\section{RECOMENDACIONES}

1. La presentación de los títulos de los proyectos de investigación, deben de expresar claramente el problema y entorno donde se ha detectado al problema. 
2. La posición filosófica a ser asumida, debe reflejar la expresión de la realidad objetiva y material, siendo obviamente optar por la posición filosófica materialista.

3. El investigador debe expresar su conocimiento especializado y el dominio cognoscitivo para la detección del problema de investigación.

4. Es concordante y lógico, expresar que la variable independiente es el problema detectado.

5. La exposición sobre la variable dependiente, en el método reflexivo exige la vinculación del investigador en un entorno.

\section{REFERENCIAS BIBLIOGRÁFICAS}

1. BERNAL TORRES, César A. (2006); Metodología de la Investigación, Colombia, Pearson Ediciones.

2. BUNGE, Mario (2000); La ciencia, su método y su filosofía, Buenos Aires, Ediciones Siglo Veinte.

3. CABALLERO ROMERO, Alejandro (2014); Metodología Integral Innovadora para Planes y Tesis. La metodología del cómo formularlo, México, CENGACE LEARNING.

4. GARCÍA CÓRDOVA, Fernando (2014); Metodología de la Investigación. Enfoque por competencias genéricas y disciplinarias, México, LIMUSA.

5. GARCÍAFERNÁNDEZ, Dora (2009); Metodología del trabajo de investigación. Guía práctica, México, Trillas.

6. HERNÁNDEZ SAMPIERI C. Roberto (2014); Metodología de la Investigación, México, McGraw-Hill.

7. ÑAUPAS, H.; MEJÍA, E.; NOVOA, E.; VILLAGÓMEZ, A.; (2014), Metodología de la Investigación, Cuantitativa-Cualitativa y redacción de la Tesis; Colombia, Ediciones de la U.

8. ROSENTAL - IUDIN (1976); Diccionario Filosófico, Buenos Aires, Editorial Universo.

9. VALARINO HERNÁNDEZ, Elizabeth (2012); Metodología de la Investigación, paso a paso, México, Trillas. 